\title{
Multiuser Detection using Hybrid ARQ with Incremental Redundancy in Overloaded MIMO Systems *
}

\author{
Muhammad Kashif ${ }^{1}$, Zakir Ullah ${ }^{2}$, Muddesar Iqbal ${ }^{3}$, Leila Musavian ${ }^{4}$, Sohail \\ Sarwar ${ }^{5}$, Xinheng Wang ${ }^{6}$, Shahid Mumtaz ${ }^{7}$, Zia Ul-Qayyum ${ }^{8}$, and Haji \\ Muhammad Safyan ${ }^{9}$ \\ ${ }^{1,2}$ Center for Advanced Studies in Engineering (CASE), Department of Electrical and \\ Computer Engg, Pakistan \\ ${ }^{3,4}$ University of Essex, School of Computer Science and Electronic Engineering, UK \\ ${ }^{5}$ National University of Sciences and Technology (NUST), SEECS, Pakistan \\ ${ }^{6}$ Department of Electrical and Electronic Engineering, Xian Jiaotong University, \\ Suzhou, China \\ ${ }^{7}$ Instituto de Telecomunicaes, Portugal \\ ${ }^{8}$ Allama Iqbal Open University, Islamabad, Pakistan \\ ${ }^{9}$ GCU, Lahore, Pakistan
}

\begin{abstract}
Multiple Input and Multiple Output (MIMO) systems use multiple antennas at both transmitter and receiver ends for increasing link capacity and spectral efficiency. However, combining schemes used for such systems face critical issues such as presence of interference, signals to interference and noise ratios (SINRs) and complexity. To overcome the asserted issues; in this paper linear multiuser detection techniques are employed in over loaded MIMO systems where the number of transmit antennas $\left(\boldsymbol{N}_{\boldsymbol{t}}\right)$ is greater than number of receiver antennas $\left(\boldsymbol{N}_{\boldsymbol{r}}\right)$, using Hybrid Automatic Repeat request with Incremental Redundancy (HARQ IR). The primary aim of this research is to enhance bit error rate (BER) and throughput by transforming an overloaded MIMO systems $\left(\boldsymbol{N}_{\boldsymbol{t}}>\boldsymbol{N}_{\boldsymbol{r}}\right)$ into critically loaded system $\left(\boldsymbol{N}_{\boldsymbol{t}}=\boldsymbol{N}_{\boldsymbol{r}}\right)$ or under loaded MIMO systems $\left(\boldsymbol{N}_{\boldsymbol{t}}<\boldsymbol{N}_{\boldsymbol{r}}\right)$ by simple retransmission method. Simulation results show unprecedented performance compared to contemporary approaches in term of throughput and BER.
\end{abstract}

Keywords: HARQ - Incremental Redundancy · Multiuser detection · MIMO

\section{Introduction}

Fifth generation (5G) systems are moving towards the concept of "device centric systems (DCS)" [1] in which many devices are transmitting and receiving at

\footnotetext{
* This work has been partially funded by the European Union Horizon 2020 research and innovation programme under the Marie Sklodowska-Curie grant agreement No.823903, (RECENT).
} 
the same time. For handling such situation, massive MIMO (Multiple Input and Multiple Output) [2] is required that deploys multiple transmitters and receivers at both ends to enhance data rate and throughput under the conditions of interference, fading and multipath. It is well known that MIMO maximizes the spectral efficiency and is widely implemented in different wireless standards such as IEEE 802.11n WLAN [3], IEEE 802.16 WiMAX [4] and LTE [5]. However, complexity and performance gets affected, especially in overloaded scenario when there are more transmitter antennas than receiver antennas, i.e. $\left(N_{t}>N_{r}\right)[6]$ [8].

In order to detect multiuser data in overloaded MIMO system, detection algorithms such as joint maximum likelihood (JML) can be used [9]. However, situation becomes more complex when transmitting antennas increases, leading it to complex situation where it is difficult to implement in practical scenario. To resolve this issue, low complex MUD (multiuser detection) techniques are proposed for overloaded conditions [6]-[8]. However, these techniques are still intricate in terms of computation and require a lot of processing to be implemented in practical scenarios.

Different hybrid automatic repeat request (HARQ) schemes are also proposed for using in conjunction with MIMO systems to further enhance throughput and reduce computational complexity. In HARQ chase combining (HARQ CC) [10] every retransmission contains the same packet i-e the data bit and parity bit while on the other hand in HARQ Incremental Redundancy (HARQ IR) [11] different information bits are transmitted from the previous one, using multiple set of coded bits. As a result these multiple coded bit results in improved performance at the receiver end. Moreover, reliability is increased by retransmitting incremental redundancy packets on requirement basis only. Similarly, Zahid et al. [12] focused on an overloaded MIMO scenario and presented schemes of converting an overloaded MIMO systems $\left(N_{t}>N_{r}\right)$ into critically loaded MIMO systems $\left(N_{t}=N_{r}\right)$ or under loaded systems $\left(N_{t}<N_{r}\right)$ by combining all retransmissions at receiver end using HARQ [13] and HARQ Chase Combining (CC) [10] techniques.

The very focus of this research is to utilize HARQ IR scheme in overloaded MIMO system for enhancing data rate, throughput and at the same time using a simple and low complex technique for detection purpose. For this purpose, a scheme is proposed considering the concept of stacking retransmission. Multiple packets are transmitted at the same time and if the packets are decoded incorrectly at the receiver end, then transmitter sends additional redundancy bits only until either decoding is succeeded or maximum number of retransmission is reached. Here, all versions of redundant bits of the same packet are combined at the receiving end. Furthermore, transmitters whose data are correctly received remain idle in the next time slot. Simulation results of the proposed scheme clearly show enhanced throughput and reduced BER as compared to existing schemes.

The rest of the paper is organized as follow; Section-II includes related study, Section III describes system model whereas proposed methodology is given in 
section-IV. Section-V is comprised of simulation analysis establishing that the proposed technique is useful in terms of BER and throughput efficiency. Finally, Section-VI concludes the paper.

\section{Related Study}

Literature survey focuses on overloaded MIMO systems. Moreover, use of suboptimal detection techniques with simplistic implementation and optimal performance is highly desired and discussed. Two of the well known linear detection techniques such as ZF [14] and MMSE [14] significantly degrade in case of overloaded systems. In [14], low complex MIMO detection schemes have been proposed but their performance and complexity increased drastically as the number of users increases. Similarly by using other detection techniques like V-BLAST algorithms [15]; due to matrix singularity fail in overloaded conditions [6]. In [15], network coding technique is used after the summation of two packets received. Thus, by using this approach error propagation issue which is vital in V-BLAST greatly reduced. However, in overloaded MIMO systems, V-BLAST schemes are not able to detect data successfully. Various other suboptimal groupwise detection techniques [6] [7] are also proposed for overloaded systems. The soft decisions that are used internally provides a good solution for detection in overloaded systems that uses block chain architecture. This method has some limitations like as we increased the group size both its computation and performance comprises. Some of the heuristic based approaches are also anticipated for overloaded systems like ant colony optimization (ACO) [16] and genetic algorithms (GA) [17].

HARQ IR is a more sophisticated protocol in terms of error detection and correction as compared to HARQ CC [18]. Recently, in [19], a linear precoder was premeditated followed by HARQ detection at the receiver end by stacking all the received vectors from all re transmission. However this scheme had some limitation that they had considered only the case of critically loaded conditions where the number of transmitters equal to the number of receivers. This concept discussed in [19] has been extended to overloaded MIMO systems in addition to critically loaded MIMO systems with the number of transmitters exceeding the number of receivers. The concept of virtual receive antenna at receiver side has been used for efficient detection of data received, analysis of the Throughput and BER in proposed model.

\section{System Model}

In this paper, an uplink Overloaded MIMO channel is considered. Multiple users are assumed which are denoted by $U$. We define $N_{t}=\sum_{u=1}^{U} N_{t}^{u}$ as the total number of transmit antennas across all users. This equation determines the total number of Tx antennas across all users. This means that that each user has a provision of single antenna for data transmission. The proposed model is presented in Fig. 1. 


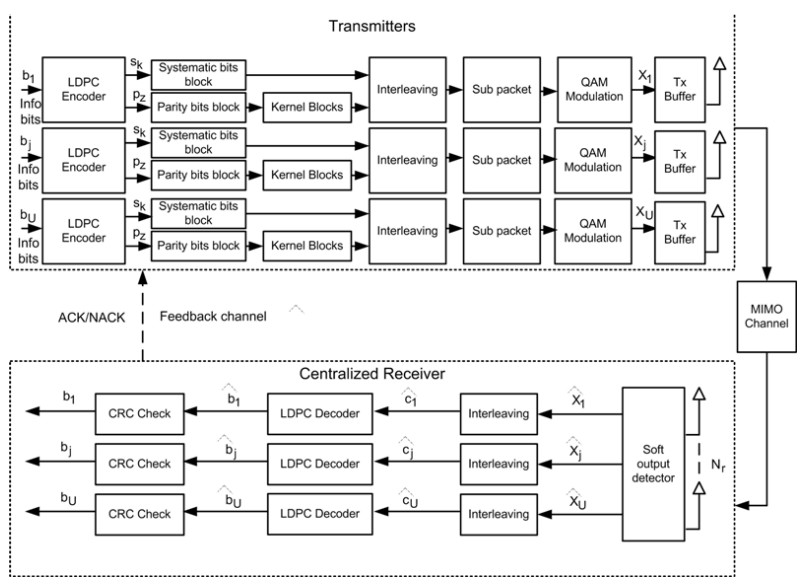

Fig. 1. HARQ IR MIMO Architecture.

In this system model, a packet constitutes of $\boldsymbol{b}_{\boldsymbol{j}}=\left(b_{(j, 1)} \ldots . . b_{(j, k)} \ldots . . b_{(j, K)}\right)$ where $K$ represents the bits which include both the info bits and CRC bits for each transmitter and $j=1,2 \ldots . . U$. The bits are then encoded by Low Density Parity Check (LDPC). LDPC block is constituted using two different kinds of block i-e Systematic and Parity bit block followed by kernel blocks. In kernel blocks different priorities are assigned related to order in which packets are transmitted. The coded bits are re ordered by interleaver to form a sub packet. Coded bits are then modulated through QAM modulation and transmitted over the wireless channel.

The received signal $\boldsymbol{y} \in \mathbb{C}^{N_{r} \times 1}$ is given by

$$
\begin{gathered}
\boldsymbol{y}=\sum_{j=1}^{N_{t}} \boldsymbol{h}_{j} \boldsymbol{x}_{j}+\boldsymbol{v}, \\
\boldsymbol{y}=\boldsymbol{H} \boldsymbol{x}+\boldsymbol{v},
\end{gathered}
$$

where $\boldsymbol{h}_{j}$ is the $j^{\text {th }}$ column of the channel matrix $\boldsymbol{H}$ of size $N_{r} \times N_{t}, \boldsymbol{x} \in \mathbb{C}^{N_{t} \times 1}$ is the overall transmitted vector and $\boldsymbol{v} \in \mathbb{C}^{N_{r} \times 1}$ is the complex Additive White Guassian Noise (AWGN). The received signal is given by

$$
\boldsymbol{Y}=\boldsymbol{H} \boldsymbol{X}+\boldsymbol{V}
$$

where $\boldsymbol{Y} \in \mathbb{C}^{N_{r} \times L}, \boldsymbol{X} \in \mathbb{C}^{N_{t} \times L}$ and $\boldsymbol{V} \in \mathbb{C}^{N_{r} \times L}$. The channel is considered as frequency-flat Rayleigh fading model. Furthermore, channel properties of a wireless communication link is known at the receiver which is well known as Channel state information (CSI).

Soft- output Multi user detection either optimal or suboptimal is used to reduce CCI due to multiple users. Following two different detection schemes have been used. 


\subsection{JML Detector}

Joint Maximum likelihood detection is the estimate of $\{\boldsymbol{x}(t)\}$ and is defined to be sequence of values which maximize the following function:

$$
\begin{gathered}
L\left(C_{p}^{u}\right)=\log \left(\frac{P\left[c_{p}^{u}=1 \mid \boldsymbol{y}, \boldsymbol{H}\right]}{P\left[c_{p}^{u}=0 \mid \boldsymbol{y}, \boldsymbol{H}\right]}\right), \\
=\log \left(\frac{\sum_{x \in X_{p}^{1}} \exp \left(-\frac{1}{\sigma_{v}^{2}}\|\boldsymbol{y}-\boldsymbol{H} \boldsymbol{x}\|^{2}\right)}{\sum_{x \in X_{p}^{0}} \exp \left(-\frac{1}{\sigma_{v}^{2}}\|\boldsymbol{y}-\boldsymbol{H} \boldsymbol{x}\|^{2}\right)}\right),
\end{gathered}
$$

where $u=1,2, \ldots U$.

Applying the maximum log approximation [23] to (5) results in

$$
L\left(C_{p}^{u}\right)=\frac{1}{\sigma_{v}^{2}}\left(\min _{x \in X_{p}^{0}}\|\boldsymbol{y}-\boldsymbol{H} \boldsymbol{x}\|^{2}-\min _{x \in X_{p}^{1}}\|\boldsymbol{y}-\boldsymbol{H} \boldsymbol{x}\|^{2}\right),
$$

However, (6) is complex to implement and its complexity increases exponentially with the increase in number of transmitting antenna.

\subsection{Suboptimal MMSE Detector}

The MMSE linear detector, however, has low complexity and yet provides acceptable performance. The received signal vector of the MMSE detector is given by

$$
\widehat{\boldsymbol{X}}_{M M S E}=\left(\boldsymbol{H}^{H} \boldsymbol{H}+\sigma_{v}^{2} \boldsymbol{I}\right)^{-1} \boldsymbol{H}^{H} \boldsymbol{y},
$$

where $\boldsymbol{I}$ is the identity matrix. The MMSE detector [22], outputs the LLR of the $p^{\text {th }}$ coded bit of the $u^{\text {th }}$ user's $j^{\text {th }}$ antenna as

$$
L\left(C_{p}^{u}, j\right)=\frac{1}{\sigma_{j, u}^{2}}\left(\min _{x \in \Omega_{p}^{0}}\left\|\widehat{x}_{j}^{u}-x\right\|^{2}-\min _{x \in \Omega_{p}^{1}}\left\|\widehat{x}_{j}^{u}-x\right\|^{2}\right),
$$

for $j=1,2, \ldots N_{t}^{u}, u=1,2, \ldots U$, where $\Omega_{p}^{0}$ and $\Omega_{p}^{1}$ represents the set of symbols in $\Omega$ whose $p^{t h}$ bit equals to 1 or $0, \widehat{x}_{j}^{u}=\frac{\left.\widehat{\boldsymbol{X}}_{M M S E}\right)_{j}^{u}}{D_{j}^{u}}$ is soft MMSE estimate of $u^{t h}$ user's $j^{t h}$ transmitted symbol, $D_{j}^{u}=\left(j+\sum_{u=1}^{u-1} N_{t}^{u}, j+\sum_{u=1}^{u-1} N_{t}^{u}\right)^{t h}$ diagonal element of $\boldsymbol{D}, \boldsymbol{D}=\left(\left[\boldsymbol{I}+\sigma_{v}^{2}\left(\boldsymbol{H}^{H} \boldsymbol{y}\right)^{-1}\right]\right)^{-1}$ and $\sigma_{j, u}^{2}=\frac{1-D_{j}^{u}}{D_{j}^{u}}$ is the noise variance.

Zahid et al. [12] in his work used the concept of HARQ CC and combined all the vectors at receiver end by considering them as a single virtual receive antenna [16]. We proposed a different approach by modifying his method and used the concept of HARQ IR in overloaded MIMO system. HARQ IR is a promising technique that has the potential of achieving enhanced performance both in terms of BER and throughput as compared to HARQ CC as also highlighted through simulation results. Secondly, HARQ IR architecture differs from HARQ $\mathrm{CC}$ in that HARQ IR needs additional signaling since the retransmission number need to be communicated to the receiver. 


\section{Proposed Scheme}

In this section, HARQ IR scheme in overloaded MIMO system using LDPC code is proposed. HARQ IR is the extended version of HARQ CC. In contrast to HARQ CC, HARQ IR retransmission is not necessarily identical to previous transmission. Instead multiple set of coded bits are generated which are a combination of multiple set of parity bits. For each negative acknowledgment from the receiver, additional parity bits are re transmitted. These multiple parity bits are different from each retransmission resulting in a higher coding and diversity gain as compared to HARQ CC. The working procedure of the proposed model is shown in Fig. 2.

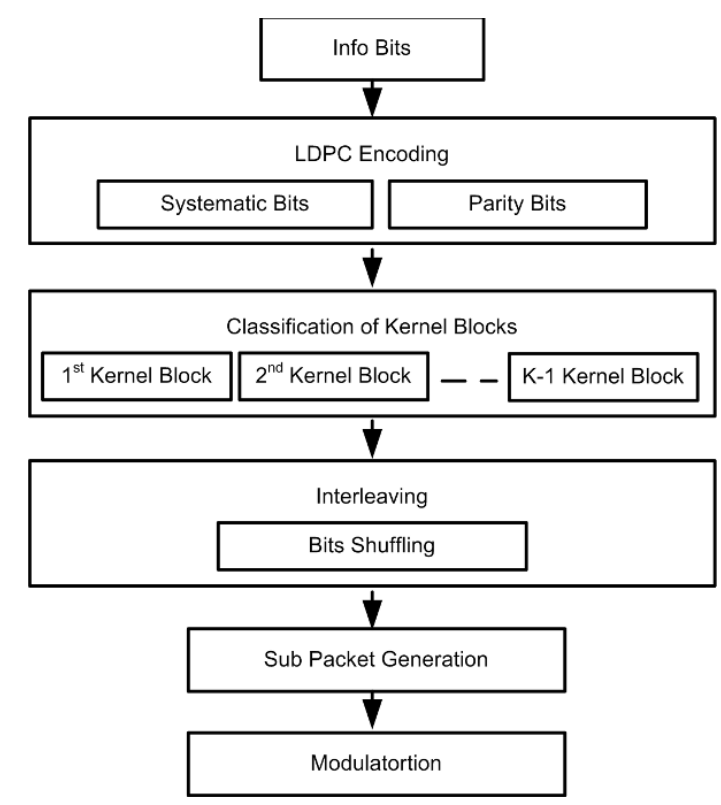

Fig. 2. Proposed HARQ IR Architecture.

Information bits encoded by LDPC encoder is given by $\boldsymbol{C}=\{\boldsymbol{S} ; \boldsymbol{P}\}=$ $\left\{s_{0}, s_{1}, \ldots s_{k} ; p_{0}, p_{1}, \ldots p_{z}\right\}$, where, $s_{0}, s_{1}, \ldots s_{k}$ are the systematic bits of $k^{t h}$ order and $p_{0}, p_{1}, \ldots p_{z}$ are the parity bits of $z^{t h}$ order. Parity bit blocks which constituted the $k^{t h}$ kernel block set $\Psi_{k}$, (for $\left.k=1,2, . .5\right)$ are given as follows:

First kernel blocks: $\Psi_{1}=\left\{P B_{1}\right\}$, where $P B_{1}$ denotes the parity bits in block 1

Second kernel blocks: $\Psi_{2}=\left\{P B_{2}\right\}$, where $P B_{2}$ denotes the parity bits in block 2

Third kernel blocks: $\Psi_{3}=\left\{P B_{3}\right\}$, where $P B_{3}$ denotes the parity bits in block 3 
Fourth kernel blocks: $\Psi_{4}=\left\{P B_{4}\right\}$, where $P B_{4}$ denotes the parity bits in block 4

Fifth kernel blocks: $\Psi_{5}=\left\{P B_{5}\right\}$, where $P B_{5}$ denotes the parity bits in block 5

The parity bits in each block are shuffled. Here, if a packet is wrongly decoded by receiver then it will send negative ACK to sender for requesting of retransmission of first kernel block. If packet is still erroneous then it will send second kernel block and this process is repeated till fifth kernel block. After fifth kernel block transmission, the packet is considered as lost if still not correctly decodable.

The interleaved encoded bits are then modulated using quadrature amplitude modulation $(\mathrm{QAM})$ as $\boldsymbol{x}^{u}=\left(x_{1}^{u}, \ldots, x_{j}^{u}, \ldots, x_{N t}^{u}\right)^{T}$ and finally transmitted employing MIMO transmission.

At receiver, soft MUD detection using either optimal JML or Linear MMSE detection is carried out with LDPC decoding. If packet is correctly received, an ACK else a NACK is sent back to sender. In case of NACK, parity bits are retransmitted incrementally until the packet is either correctly decodable and positive acknowledgment is sent or maximum limit of retransmissions is reached.

Utilizing (2), the received signal at the $g^{t h}$ (re)transmission can be written as given in

$$
\boldsymbol{y}(g)=\boldsymbol{H}(g) \boldsymbol{x}(g)+\boldsymbol{v}(g),
$$

where $g=1,2, \ldots G$ is the retransmission number.

Similarly, the stacked received vectors [21] after $G$ transmissions can be written as

$$
\boldsymbol{r}=\left[\begin{array}{c}
\boldsymbol{y}(1) \\
\boldsymbol{y}(2) \\
\cdot \\
\boldsymbol{y}(g)
\end{array}\right]=\left[\begin{array}{c}
\boldsymbol{H}(1) \\
\boldsymbol{H}(2) \\
\cdot \\
\cdot \\
\boldsymbol{H}(g)
\end{array}\right] \boldsymbol{x}+\left[\begin{array}{c}
\boldsymbol{v}(1) \\
\boldsymbol{v}(2) \\
\cdot \\
\boldsymbol{v}(g)
\end{array}\right]
$$

One of the major drawbacks of (10) is the wastage of resources (in terms of link bandwidth, transmitting power and resource allocation at the receiver end). This wastage of resources is caused by retransmission of same data during all retransmissions. To cater for this situation i-e to avoid multiple retransmission of the same packet, Scheme HARQ IR is proposed as explained in following section.

\subsection{HARQ IR Stacking}

Consider a case of over loaded MIMO system, i.e., $N_{t}>N_{r}$. In this Scheme, at first transmission, i.e., at time $t=1$, all users send their packets. Transmitters whose packets are correctly received remain idle in the next time slot, whereas the transmitters of erroneous packets send their packets again in next retransmission. This method has an added advantage that it reduces co-channel interference and hence improves throughput. Co-channel interference is reduced due to the fact 
that those users whose data is correctly received are not available in next time slot and hence result in reduced system loading

The received signal vector from erroneous user is given by

$$
\overline{\boldsymbol{y}}(1)=\boldsymbol{y}(1)-\sum_{u=1}^{U} \boldsymbol{H}(1) \boldsymbol{x}(1) .
$$

Similarly, during second time interval, the correctly decoded users remain silent while the erroneous decoded ones are requested for re-transmission. This re-transmission is carried out by incrementally transmitting parity bits of kernel blocks. Here, the received signal vector is given as

$$
\overline{\boldsymbol{y}}(2)=\overline{\boldsymbol{H}}(2) \boldsymbol{x}(2)+\boldsymbol{v}(2) .
$$

After this, received signal vectors, i.e., $\overline{\boldsymbol{y}}(1)$ and $\overline{\boldsymbol{y}}(2)$ are stacked together for creating virtual receive antenna, as given by

$$
\boldsymbol{r}=\left[\begin{array}{c}
\overline{\boldsymbol{y}}(1) \\
\overline{\boldsymbol{y}}(2)
\end{array}\right] .
$$

Now, MUD is employed on vector $\boldsymbol{r}$ for generating soft estimates as decoder's input. This process continues till the transmission of parity bits of last kernel block. Moreover, packet is considered lost if maximum $G$ is reached and correct decoding is not resulted.

\section{Performance Analysis}

The proposed scheme is evaluated in this section and compared with HARQ CC [12] in order to confirm its effectiveness using bit error rate (BER) and throughput as performance parameters in various simulations scenarios. In simulations, four users are considered. For packet encoding, LDPC code is used with the rate of $R_{c}=1 / 2$. Similarly, 16-QAM is used as modulation whereas both JML and linear MMSE are used as detectors. The default simulation parameters are enlisted in Table (1).

\subsection{Bit Error Rate (BER) Analysis}

For a 4x1 Over loaded MIMO system (i.e., four transmitters and single receiver), employing soft output detection scheme (JML and MMSE) and using BER performance as a parameter is presented in Fig. 3 and Fig. 4. Fig. 3 shows BER performance of HARQ IR and HARQ CC using JML detection during $G=2$, 3 and 4, respectively. JML detection compares received signal with all possible transmitted signal vectors and then estimates the transmitted symbol vector which is modified by channel matrix $\boldsymbol{H}$. In the proposed scheme, as shown in Fig. 3, it is clearly observed that HARQ IR outperforms HARQ CC for each transmission and lower BER is achieved when $G$ is 2,3 and 4 . For each case of 
Table 1. Default simulation parameters

\begin{tabular}{|c|c|}
\hline Parameter & Value \\
\hline Number of transmitters $\left(N_{t}\right)$ & 4 \\
\hline Number of receivers $\left(N_{r}\right)$ & 1 \\
\hline Number of retransmission $(G)$ & 4 \\
\hline Modulation type & 16 -QAM \\
\hline Channel model & Uplink \\
\hline Packet size (Info bits + CRC) & 576 \\
\hline Encoder & LDPC \\
\hline Encoder rate $\left(R_{c}\right)$ & $1 / 2$ \\
\hline Number of kernel blocks & 5 \\
\hline
\end{tabular}

retransmission at different $\mathrm{SNR}(\mathrm{Eb} / \mathrm{No}(\mathrm{dB}))$, BER of HARQ IR is less than BER of HARQ CC. This means that fewer bits are found in error in HARQ IR than in HARQ CC. Furthermore, it is also attested that BER decreases with the increase in SNR. Moreover, in proposed scheme, co-channel interference is reduced resulting in system reduction i-e shifting of system from overloaded to under loaded or critically loaded. When compared the HARQ IR with HARQ CC, HARQ CC retransmits the same message in each retransmission while HARQ IR has an advantage that if message is not correctly decoded it requests only for retransmission of kernel blocks incrementally. This incremental retransmission results in improved BER. Furthermore, BER is also improved with increasing the diversity order. Also as shown, when $G$ is 4 , enhanced performance of the scheme is observed as compared to the performance when $G$ is 2 and 3 .

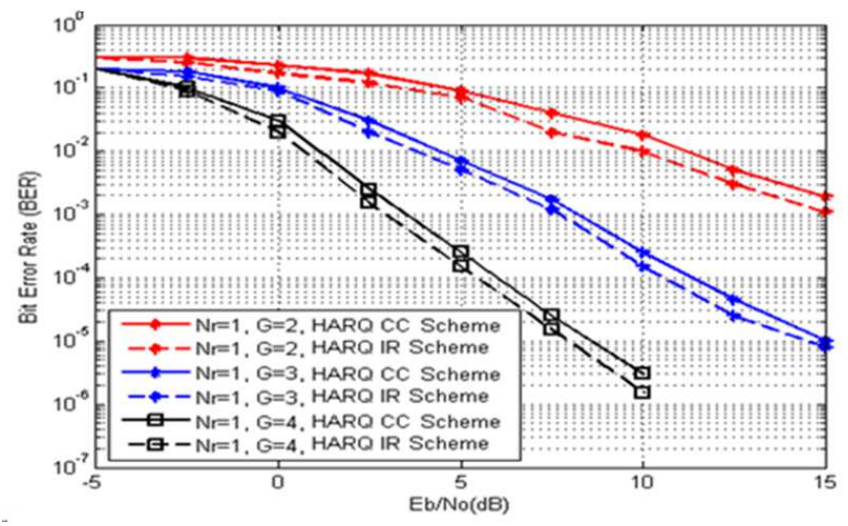

Fig. 3. BER comparison of HARQ IR and HARQ CC with JML detection. 
Fig. 4 shows BER performance of HARQ IR and HARQ CC using the Scheme and with linear MMSE during $G=2,3$ and 4 respectively. Here also, HARQ IR attested improved performance in comparison to HARQ CC. Moreover, in terms of spectral efficiency HARQ IR perform better than HARQ CC due to the fact that HARQ IR adapts its error correcting code redundancy to varying channel conditions. MMSE detection mitigates the effect of noise by taking into account the interference from other symbols and noise. Higher values of $G$ means higher number of retransmission and more reduction of system loading due to reduction in correctly decoded users. Hence resulting in improved SNR.

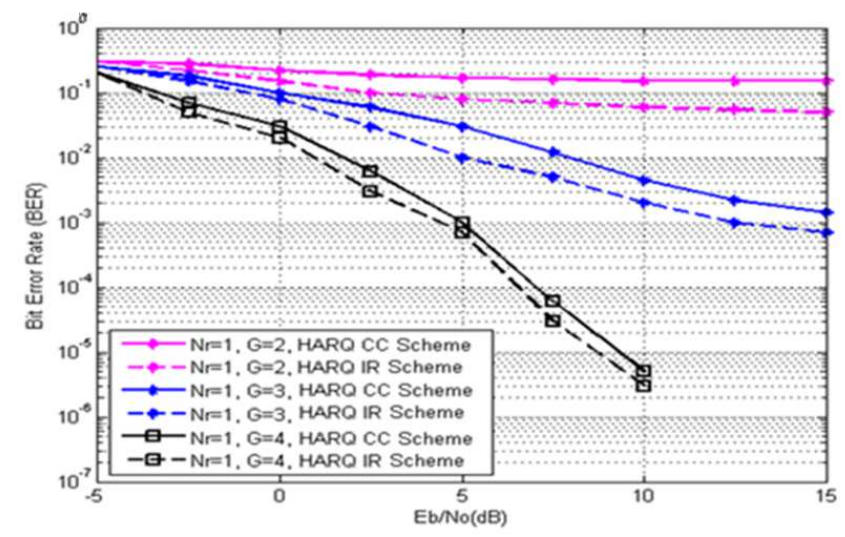

Fig. 4. BER comparison of HARQ IR and HARQ CC with MMSE detection.

\subsection{Throughput Analysis}

The most important aspect of this work is to improve throughput especially in noisy wireless channel. It is calculated for each retransmission using (14).

$$
\delta=\frac{\log _{2}\left(\omega R\left(1-P_{\text {rate }}\right)\right)}{N_{\text {avg }}}(b / s / H z),
$$

where $N_{\text {avg }}$ is the maximum number of retransmission, $P_{\text {rate }}$ is drop packet rate and $R$ is the code rate. Fig. 5 shows throughput comparison of HARQ IR with HARQ CC when $G$ is 4 . Throughput analysis is achieved by implementing both the detection schemes, i.e., JML and MMSE in the proposed scheme.

As shown in Fig. 5, HARQ IR throughput is much better than HARQ CC. For example, at SNR of $10 \mathrm{~Eb} / \mathrm{No}(\mathrm{dB})$, throughput of Scheme HARQ IR proposed is 0.75 as compared to HARQ CC which is 0.45 with JML detection, it means throughput efficiency is improved by $20 \%$ for HARQ IR as compared to HARQ CC. Similarly, for MMSE detection throughput of HARQ IR is 0.46 in contrast 


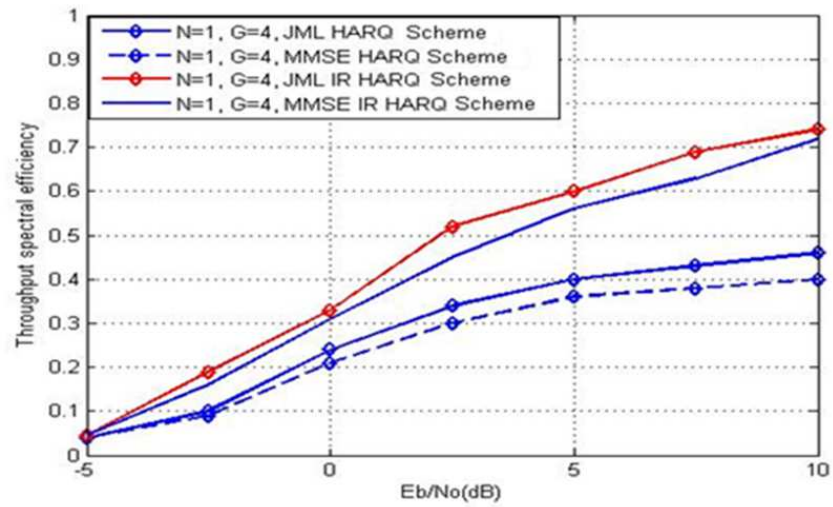

Fig. 5. Throughput comparison of HARQ IR and HARQ CC.

to HARQ CC which is 0.4 . This improved performance of HARQ IR is due to the fact that instead of retransmitting whole packet as practiced in HARQ CC, it retransmits additional parity bits only.

\section{Conclusion}

In this paper, a detection scheme has been implemented with the combination of HARQ IR in overloaded MIMO system in which through simple retransmission, overloaded MIMO system is reduced to critically or under loaded MIMO system. This scheme help in using linear MUD algorithms instead of using complex MUD set of rules. Performance of the scheme is evaluated using BER and throughput and compared with HARQ CC and better results are achieved with the proposed scheme by adding no complexity to the system. Hence, the approach may be considered to be used in LTE and other advanced wireless systems.

For our future work as we observe in this paper, an error free feedback with perfect channel state information is assumed which is practically impossible. Moreover, CRC detection is considered as error free, we will consider both these factors as well in our future work.

\section{References}

1. M. Z. Shakir, M. Ismail, X. Wang, K. A. Qaraqe and E. Serpedin, "From D2D to Ds2D: Prolonging the Battery Life of Mobile Devices via Ds2D Communications," IEEE Wirel. Commun., vol. 24, no.4, pp. 55-63, August. 2017.

2. E. G. Larsson and L. Van der Perre, "Massive MIMO for 5G," IEEE 5G Tech Focus, vol. 1, no. 1, 2017.

3. M. Uthansakul and P. Uthansakul, "Experiments with a low-profile beamforming MIMO system for WLAN applications," IEEE Antennas and Propagation Mag., vol. 53, no. 6 , pp. 56-69, Dec. 2011. 
4. T. Siadari, F. Rezha and S.-Y. Shin, "Applied MIMO based on Hadamard transform in IEEE 802.16 WiMAX system," IET Int'l. Conf. on Inform. and Commun. Tech. (IETICT), pp. 582-586, April 2013.

5. B. Badic, R. Balraj, T. Scholand, Z. Bai and S. Iwelski, "Impact of feedback and user pairing schemes on receiver performance in MU-MIMO LTE systems," IEEE Wireless Commun. and Networking Conf. (WCNC), pp. 399-403, April 2012.

6. B. W. Zarikoff, J. K. Cavers and S. Bavarian, "An Iterative groupwise multiuser detector for overloaded MIMO applications," IEEE Trans. Wireless Commun., vol. 6, no. 2, pp. 443-447, Feb. 2007.

7. M. Krause, D. P. Taylor and P. A. Martin, "List-based group-wise symbol detection for multiple signal communications," IEEE Trans. Wireless. Commun., vol. 10, no. 5, pp. 1636-1644, May 2011.

8. G. Colman and T. J. Willink, "Overloaded array processing using genetic algorithms with soft-biased initialization," IEEE Trans. Veh. Tech., vol. 57, no. 4, pp. 2123-2131, July 2008.

9. S. J. Grant and J. K. Cavers, "Performance enhancement through joint detection of co channel signals using diversity arrays," IEEE Trans. Commun., vol. 46, no. 8, pp. 1038-1049, August 1998.

10. D. Chase, "Code combining-a maximum likelihood decoding approach for combining an arbitrary number of noisy packets," IEEE Trans. Commun., vol. 33, pp. 385-393, May 1985.

11. $\mathrm{Y} . \mathrm{Wu}$ and $\mathrm{S} . \mathrm{Xu}$, "Energy-efficient multi-user resource management with IRHARQ," IEEE 75th Vehicular Technology Conference (VTC Spring), pp. 1-5, May 2012.

12. Z. Rauf, P. A. Martin, and D. P. Taylor, "Multiuser detection of overloaded systems employing HARQ," IEEE International Conference on Communication Systems (ICCS), pp. 300-304, Nov. 2012.

13. S. Lin and D. J. Costello, Error Control Coding, 2nd ed. Prentice Hall, 2004

14. L. Bai and J. Choi, Low Complexity MIMO Detection, Springer, 2012.

15. S. Zhang, C. Nie, L. Lu, S. Zhang, and G. Qian, "MIMO physical layer network coding based on VBLAST detection," IEEE Int'l. Conf. on Wireless Comm. \& Sig. Proc. (WCSP), pp. 1-5, Oct. 2012.

16. K. T. Tasneem, P. A. Martin, and D. P. Taylor, "Iterative soft detection of cochannel signals using ant colony optimization," IEEE Int'l. Symp. on Personal, Indoor and Mobile Radio Comm. (PIMRC), pp. 1617-1621, Sep. 2012.

17. K. Obaidullah and Y. Miyanaga, "Efficient algorithm with lognormal distributions for overloaded MIMO wireless system," IEEE Sig. and Info.Process Assoc Annual Summit and Conf. (APSIPA ASC), pp. 1-4, Dec. 2012.

18. I. Stanojev, O. Simeone, Y. Bar-Ness, and D. H. Kim, "Energy efficiency of noncollaborative and collaborative hybrid-ARQ protocols," IEEE Trans. Wirel. Commun., vol. 8, no. 1, pp. 326-335, 2009.

19. X. Liang, C. Zhao and Z. Ding, "Sequential linear MIMO precoder optimization for Hybrid ARQ retransmission of QAM signals," IEEE Commun. Letters., vol. 15, no. 9, pp. 913-915, Sep. 2011.

20. P. Larsson and N. Johansson, "Multi-User ARQ," IEEE Vehicular Technology Conference (VTC 2006-Spring), Melbourne, Australia, May 2006.

21. P. Larsson, "Multicast Multiuser ARQ," IEEE Wireless Communications and Networking Conference (WCNC08), Las Vegas, USA, April 2008.

22. P. FertI, J. JalMn and G. Matz, "Capacity-based performance comparison of MIMO-BICM demodulators," SPAWC, pp. 166-170, 2008. 
23. S. H. Miiller-Weinfurther, "Coding approaches for multiple antenna transmission in fast fading and OFDM," IEEE Trans. Signal Proc., vol. 50, no. 10, pp. 2442-2450, Oct. 2002 . 